\title{
Talaromyces marneffei Infection in Systemic Lupus Erythematosus Patients: Report of Two Cases and Review of the Literature
}

\author{
Jingshan Wei ${ }^{1}$ \\ Ye Qiu' \\ Wen Zeng' \\ Mianluan Pan' \\ Jianquan Zhang ${ }^{1,2}$ \\ 'Department of Respiratory and Critical \\ Care Medicine, The First Affiliated \\ Hospital of Guangxi Medical University, \\ Nanning, Guangxi 53002I, Pepole's \\ Republic of China; ${ }^{2}$ Department of \\ Respiratory and Critical Care Medicine, \\ The Eighth Affiliated Hospital of Sun Yat- \\ Sen University, Shenzhen, Guangdong \\ 518033, Pepole's Republic of China
}

This article was published in the following Dove Press journal: Infection and Drug Resistance

Purpose: Talaromyces marneffei is a highly invasive fungus, causing fatal mycosis in patients with or without HIV in Southeast and Eastern Asia. However, its presence in patients with systemic lupus erythematosus is rarely reported.

Methods: We reported two SLE patients infected by T. marneffei and reviewed other patients reported in the English literature. All cases were pooled for analysis.

Results: Eleven patients with SLE infected with T. marneffei infection were identified, including the two presented here. Three were male and eight were female; all were HIV negative. All the patients, except two where data were missing, had received immunosuppressants before T. marneffei infection. The main clinical features included fever, cough, lymph node enlargement, gastrointestinal symptoms, and rash. Five patients were misdiagnosed as having SLE exacerbation. T. marneffei was detected via culture or histopathologic analysis, with the fungus most commonly found in the blood. Seven of the 11 patients were successfully treated by timely antifungal therapy with concomitant SLE control, while four patients who did not receive antifungal therapy died.

Conclusion: T. marneffei infection should be excluded when SLE patients, especially if on long-term immunosuppressants, present with fever, cough, lymph node enlargement, gastrointestinal symptoms, and rash. Controlling the lupus and timely antifungal treatment can improve the outcomes of SLE patients with T. marneffei infection.

Keywords: Talaromyces marneffei, systemic lupus erythematosus, immunosuppressant

\section{Introduction}

Systemic lupus erythematosus (SLE) is a chronic autoimmune disease involving multiple systems and is characterized by pathogenic autoantibodies and uncontrolled inflammatory response. ${ }^{1}$ Intrinsic immune system defects combined with immunosuppressive therapy predispose SLE patients to various infections, including bacterial, fungal, parasitic, and viral infections. ${ }^{1-4}$

Talaromyces marneffei is a highly invasive fungus endemic to Southeast and Eastern Asia. 5,6 It commonly occurs in human immunodeficiency virus (HIV)positive patients, but has been reported in both immunocompetent and immunocompromised HIV-negative patients. ${ }^{7,8}$ Reports of its presence in SLE patients are rare, ${ }^{9-15}$ and risk factors remain unclear. The diagnosis is challenging owing to nonspecific manifestations that are similar to those of active SLE.

Here, we report two SLE patients infected with $T$. marneffei and review the literature with a focus on risk factors, clinical characteristics, treatments, and
Correspondence: Jianquan Zhang Department of Respiratory and Critical Care Medicine, The Eighth Affiliated Hospital of Sun Yat-Sen University, Shenzhen, Guangdong 5I8033, Pepole's Republic of China

$\mathrm{Tel}+86 \quad 13978123845$

Fax +8607715350031

Email jqzhang2002@I26.com
Infection and Drug Resistance 2020:13 381I-3816 
outcomes. We hope this article will serve as a reference for its early diagnosis and treatment.

\section{Methods}

We reported two SLE patients who had been diagnosed with T. marneffei infection at the First Affiliated Hospital of Guangxi Medical University. Other reported cases of T. marneffei infection in SLE patients were identified by searching the PubMed, Wanfang, and Google Scholar databases up to June 2020 using the keywords "Penicillium marneffei or Penicilliosis marneffei or Talaromyces marneffei" and "Systemic lupus erythematosus or SLE." Only articles published in the English language were selected. The retrieved cases were pooled with the two cases in this report for analysis.

\section{Results}

\section{Case I}

A 49-year-old Chinese man, who had a 2-month history of SLE and treated with oral prednisone $(30 \mathrm{mg} /$ day), was admitted to a local hospital in February 2017 due to repeated fever, cough and fatigue. He was a farmer living in Guangxi. During hospitalization, Gram-negative bacteria were isolated from his blood culture and antibiotic (meropenem) for septicemia administered during a period of 3 weeks without improvement. He was then referred to our hospital for further treatment.

Upon admission to our hospital, physical examination showed annular erythema of the inner canthus and eyelids, a mouth ulcer and massive ecchymosis of the forearms. Routine blood examination revealed the following: white blood cells, $3.65 \times 10^{9} / \mathrm{L}$ with $81.4 \%$ neutrophils; hemoglobin, $85.80 \mathrm{~g} / \mathrm{L}$; and platelets, $649.20 \times 109 / \mathrm{L}$. The c-reactive protein (CRP) level $(38.99 \mathrm{mg} / \mathrm{L})$ and erythrocyte sedimentation rate ( $>140 \mathrm{~mm} /$ hour) were elevated. The 24-hour urine protein level was $2655.0 \mathrm{mg}$, anti-dsDNA antibody titers were normal, and serum complements were low (C3, $0.519 \mathrm{~g} / \mathrm{l} ; \mathrm{C} 4,0.125 \mathrm{~g} / \mathrm{l})$. Assessment for anti-nuclear antibody and anti-SS-A indicated positivity while anti-HIV antibody was not detected. The liver functions (alanine aminotransferase, $144 \mathrm{U} / \mathrm{L}$; aspartate aminotransferase $677 \mathrm{U} / \mathrm{L}$ ) were deranged, and blood coagulation (thrombin time $>120 \mathrm{~s}$, fibrinogen $0.69 \mathrm{~g} / \mathrm{l})$ was impaired. The CD4+ T-lymphocyte count ( 95 cells $/ \mu \mathrm{L}$ ) was lower than normal. A sputum smear contained hyphae. Chest computed tomography (CT) scan revealed infiltration in the upper lobes of the lungs, swollen mediastinal lymph nodes, and a slight pleural effusion. A diagnosis of active SLE with lupus nephritis, pneumonia, and septicemia was established.

He received intravenous methylprednisolone ( $80 \mathrm{mg} /$ day), empirical intravenous antibacterial (meropenem $1 \mathrm{gq} 8 \mathrm{~h}$ ), and antifungal (fluconazole $200 \mathrm{mg} /$ day). His temperature subsequently returned to normal. However, his platelet count progressively declined to $42.4 \times 10^{9} / \mathrm{L}$ and he developed hemoptysis. Nine days post-admission, chest CT showed increased infiltration and pleural effusion. Finally, the sputum and bone marrow cultures were positive for T. marneffei.

Methylprednisolone was changed to prednisone $50 \mathrm{mg}$ /day. Intravenous amphotericin B (5 mg/day gradually increased to $25 \mathrm{mg} /$ day) and oral voriconazole ( $400 \mathrm{mg} /$ day) were administered for 2 weeks, followed by oral itraconazole ( $400 \mathrm{mg} /$ day) for 11 months. His condition improved and there was no recurrence of $T$. marneffei infection at the 3-year follow-up.

\section{Case 2}

A 27-year-old Chinese woman, who had received oral prednisone (30 mg/day) 4 years for SLE, was admitted to our hospital in July 2003 because of thigh swelling, pain, and fever for 3 weeks. She was a worker and born in Guangxi. On admission, her temperature was $38.8^{\circ} \mathrm{C}$, and she had large scattered patches of edematous erythema on her swollen right thigh. Laboratory tests revealed the following: white blood cell count, $8.93 \times 10^{9} / \mathrm{L}$ with $94.8 \%$ neutrophils; hemoglobin, $61 \mathrm{~g} / \mathrm{L}$; erythrocyte sedimentation rate, $138 \mathrm{~mm} /$ hour; a high CRP level $(46.8 \mathrm{mg} / \mathrm{mL})$; normal serum complements and anti-dsDNA antibody titers. Antinuclear antibodies were highly expressed (109.4 IU/mL), whereas anti-HIV antibodies were absent. Blood cultures were positive for coagulase-negative Staphylococcus. The histopathology of the skin lesions indicated vasculitis, which is consistent with SLE. Active SLE and septicemia were diagnosed. She responded to dexamethasone $(10 \mathrm{mg} /$ day) and antibiotics (levofloxacin and cefpiramide) and was discharged after 2 weeks of treatment.

In October 2003, she was readmitted to our hospital with recurrent swelling of her right thigh and cutaneous ulcers. The ulcer on her right thigh was $3 \mathrm{~cm}$ in diameter with purulent secretions. Her laboratory test results in the current admission were similar to those in July 2003. T. marneffei was identified in cultures prepared from 
blood and ulcer secretions, and coagulase-negative Staphylococcus aureus was isolated from ulcer secretions.

She received oral prednisone (40 $\mathrm{mg} /$ day), broadspectrum antibiotics (intravenous cefuroxime and clindamycin), and intravenous fluconazole (300 mg/day). One month later, her clinical symptoms had significantly improved; fluconazole was reduced to $200 \mathrm{mg} / \mathrm{day}$, and the antibiotics were discontinued. She was discharged 3 months after treatment and oral fluconazole (200 mg/day) was administered for an additional 6 months. There was no recurrence at the 1-year follow up.

\section{Literature Review}

Including our cases, 11 cases of SLE with T. marneffei infection have been reported in the English literature; their characteristics are detailed in Table 1. All patients were HIV negative and from southern China or Southeast Asia; three were male and eight were female, and the average age was 37.18 (range, 23-65) years. All patients presented with fever; four had cough, lymph node enlargement, and/or gastrointestinal symptoms and two had rashes. The duration of SLE ranged from 2 months to 24 years.

Chronic immunosuppression preceded T. marneffei infection in nine cases; data were unavailable for the remaining two cases. All nine patients had received prednisone prior to infection, with three concomitantly receiving additional immunosuppressants (eg, mycophenolate mofetil, azathioprine, or hydroxychloroquine). All patients with CRP data $(n=7)$ had an elevated level. Five patients including ours were initially misdiagnosed with SLE exacerbation, leading to delayed treatment of the infection and consequent increase of the immunosuppressant dosage and eventual worsening of the condition.

T. marneffei infection was diagnosed via culture or histopathologic examination, with the fungus most commonly found in the blood (five patients). Seven patients were expeditiously treated with antifungal drugs after T. marneffei infection was confirmed. The duration of the antifungal treatment was $12-80$ weeks, with continuous control of the SLE. The treatment eliminated the infection in these patients. The four patients who did not receive antifungal therapy died. One died from multiple organ failure, while the second patient died from bleeding and disseminated intravascular coagulation. Data on the cause of mortality were not available for the remaining two patients.

\section{Discussion}

T. marneffei can invade various organs in HIV-negative patients, including lungs, skin and lymph nodes. ${ }^{7,14}$ The common clinical manifestations of infection are fever, malaise, weight loss, skin and soft tissue lesions, hepatosplenomegaly, lymphadenopathy, cough, and dyspnea. ${ }^{7}$ These manifestations are shared by SLE, which involves multiple organs; and fever is a typical symptom of an SLE flare-up. ${ }^{1}$ Consequently, diagnosing T. marneffei infections in SLE patients is challenging.

Of the 11 reported SLE patients infected with T. marneffei, fever, cough, lymph node enlargement, gastrointestinal symptoms and rashes were the most common manifestations. Among them, five patients were inaccurately diagnosed with SLE exacerbations resulting in treatment with increasing dosages of immunosuppressants. Increasing the dosage of immunosuppressant before excluding T. marneffei infection results in dissemination of the infection and is fatal. Hence, clinicians should be aware of this uncommon fungus and pay close attention to the results of microbiological, pathological, and laboratory investigations. Doing so will allow them to distinguish SLE activity from T. marneffei infection and devise appropriate treatment plans. CRP level is a good marker to distinguish between infection and active SLE; it has high sensitivity and specificity, and an increase level in CRP suggests a combined infection in patients with SLE. ${ }^{1-3}$ Kim et al demonstrated in a ROC analysis of CRP that the area under the curve was 0.966 (95\% CI 0.925-1.007), and CRP levels higher than $1.35 \mathrm{mg} / \mathrm{dL}$ indicated infection in SLE patients with a sensitivity of $100 \%$ and a specificity of $90 \%{ }^{16}$ Seven of the reported SLE patients with T. marneffei infection had available CRP data, and CRP levels in all seven were elevated and higher than $1.35 \mathrm{mg} /$ dL. Definitive diagnosis of T. marneffei infection relies on positive cultures and microscopy from a variety of clinical specimens. T. marneffei is a dimorphic fungus: it has a yeast form at $37^{\circ} \mathrm{C}$ and a mycelial form with a characteristic diffusible red pigment at $25^{\circ} \mathrm{C}^{17}$ The yeast form is visualized using the periodic acid-Schiff stain or the Wright stain, which reveals yeast-like or sausage-like cells $2-3 \mathrm{~mm}$ in diameter with a central transverse septum. ${ }^{8}$ Acid fast staining and other specific staining procedures are helpful for differentiating T. marneffei from other pathogens.

A previous study showed that prolonged immunosuppression renders patients more susceptible to opportunistic 


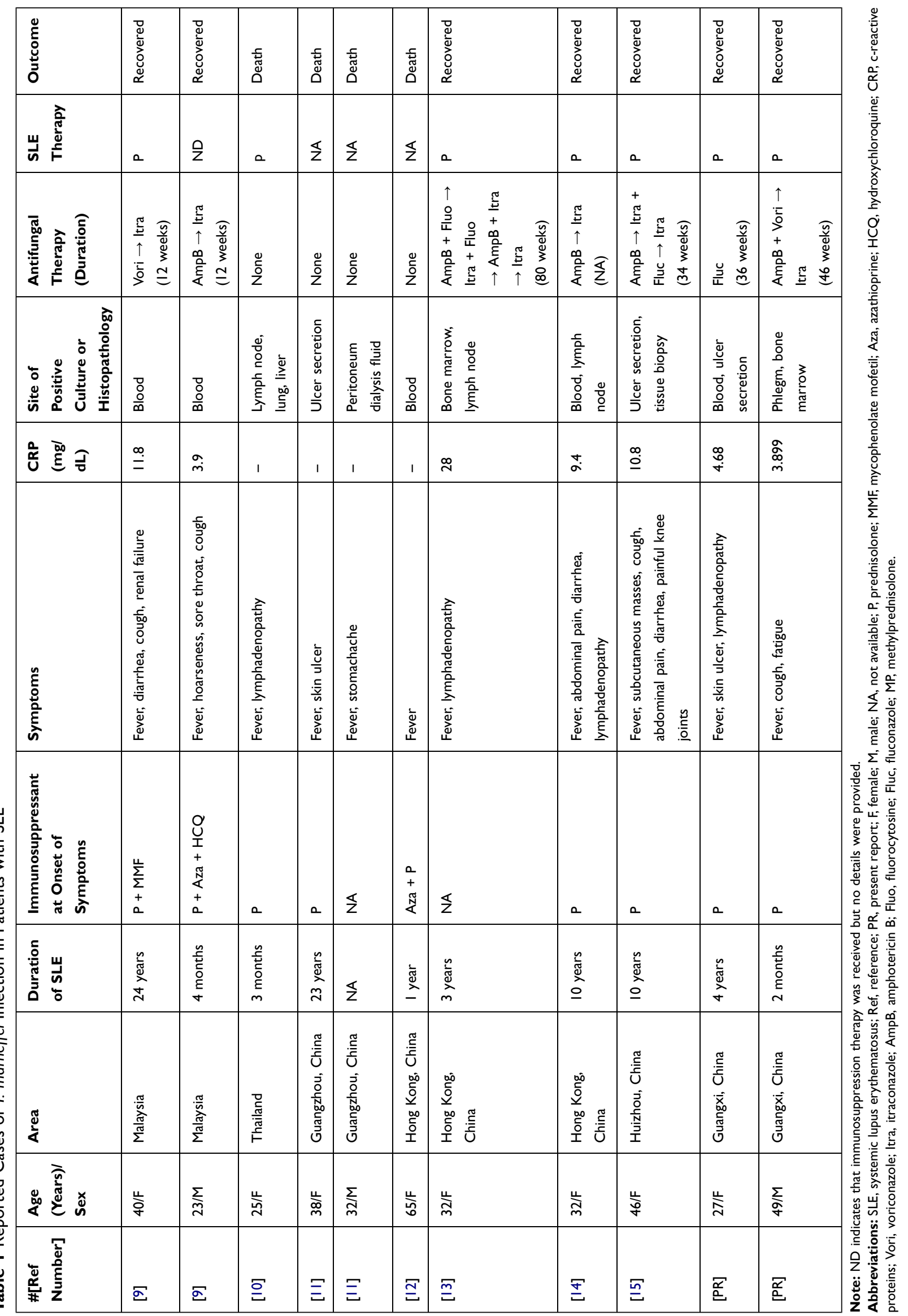


infections. ${ }^{18}$ All nine reported T. marneffei-infected SLE patients for whom immunosuppression data were available had undergone chronic immunosuppression before infection. Hence, long-term use of immunosuppressants may be an important risk factor for $T$. marneffei infection in patients with SLE. However, more cases are needed to conclusively identify the relevant risk factors.

T. marneffei infection is associated with high mortality rates, and early administration of antifungal therapy can improve prognosis. ${ }^{8,17}$ Agents that effectively treat T. marneffei infection include voriconazole, itraconazole, amphotericin B, 5-flucytosine, and fluconazole. ${ }^{17,19}$ The most commonly recommended antifungal therapy is amphotericin B combined with itraconazole., ${ }^{9,20}$ Four of the $11(36.4 \%)$ reported T. marneffei-infected SLE patients died without receiving antifungal therapy. Among them, one patient refused to use antifungal drugs, one patient was not treated with antifungal agents since the diagnosis was made posthumously, and the remaining two did not have data available. The remaining seven patients were treated using a timely antifungal therapy and concomitant control of the lupus; most of them initially received amphotericin B-based antifungal drugs followed by itraconazole. All of them were cured clinically. These findings indicate timely, effective antifungal therapy, combined with control of the lupus are important for T. marneffei infection in SLE patients. There is currently no standard treatment duration for T. marneffei infection in HIV-negative individuals. T. marneffei infection readily relapses regardless of immune system status, and there are some reports of relapse after many years. ${ }^{8,17}$ Hence, for SLE patients who require chronic immunosuppression, long-term antifungal therapy and follow up may be necessary. The key indicators of when to stop antifungal treatment include clinical manifestation, imaging results and laboratory examination.

\section{Conclusion}

Clinicians should be aware of T. marneffei infection when SLE patients, especially those on long-term immunosuppressants, present with fever, cough, lymph node enlargement, gastrointestinal symptoms, and rash. Early diagnosis and antifungal therapy combined with control of lupus symptoms is critical for a favorable outcome.

\section{Ethical Approval}

Written informed consent was obtained from the patients for publication of this report. Copies the written consent are available for review by the Editor of this journal. This study was approved by the ethics committee associated with the Faculty of Medicine at The First Affiliated Hospital of Guangxi Medical University.

\section{Acknowledgments}

All authors thank Editage for English language editing.

\section{Author Contributions}

All authors made a significant contribution to the work reported, whether that is in the conception, study design, execution, acquisition of data, analysis and interpretation, or in all these areas; took part in drafting, revising or critically reviewing the article; gave final approval of the version to be published; have agreed on the journal to which the article has been submitted; and agree to be accountable for all aspects of the work.

\section{Funding}

This work was supported by the National Natural Science Foundation of China [grant nos. 81760010].

\section{Disclosure}

The authors declare that they have no competing interests. The abstract of this paper was presented at the American Thoracic Society International Conference as a poster presentation

\section{References}

1. Jung JY, Suh CH. Infection in systemic lupus erythematosus, similarities, and differences with lupus flare. Korean J Intern Med. 2017;32 (3):429-438. doi:10.3904/kjim.2016.234

2. Sciascia S, Ceberio L, Garcia-Fernandez C, Roccatello D, Karim Y, Cuadrado MJ. Systemic lupus erythematosus and infections: clinical importance of conventional and upcoming biomarkers. Autoimmun Rev. 2012;12(2):157-163. doi:10.1016/j.autrev.2012.03.009

3. Wang J, Niu R, Jiang L, et al. The diagnostic values of C-reactive protein and procalcitonin in identifying systemic lupus erythematosus infection and disease activity. Medicine (Baltimore). 2019;98(33): e16798. doi:10.1097/MD.0000000000016798

4. Caza T, Oaks Z, Perl A. Interplay of infections, autoimmunity, and immunosuppression in systemic lupus erythematosus. Int Rev Immunol. 2014;33(4):330-363. doi:10.3109/08830185.2013.863305

5. Chakrabarti A, Slavin MA. Endemic fungal infections in the Asia-Pacific region. Med Mycol. 2011;49(4):337-344. doi:10.3109/ 13693786.2010 .551426

6. Jiang J, Meng S, Huang S, et al. Effects of Talaromyces marneffei infection on mortality of HIV/AIDS patients in southern China: a retrospective cohort study. Clin Microbiol Infect. 2019;25 (2):233-241. doi:10.1016/j.cmi.2018.04.018

7. Chan JF, Lau SK, Yuen KY, Woo PC. Talaromyces (Penicillium) marneffei infection in non-HIV-infected patients. Emerg Microbes Infect. 2016;5:e19. doi:10.1038/emi.2016.18 
8. Qiu Y, Liao H, Zhang J, Zhong X, Tan C, Lu D. Differences in clinical characteristics and prognosis of Penicilliosis among HIV-negative patients with or without underlying disease in Southern China: a retrospective study. BMC Infect Dis. 2015;15 (1):525. doi:10.1186/s12879-015-1243-y

9. Chong YB, Tan LP, Robinson S, et al. Penicilliosis in lupus patients presenting with unresolved fever: a report of 2 cases and literature review. Trop Biomed. 2012;29(2):270-276.

10. Jayanetra P, Nitiyanant P, Ajello L, et al. Penicilliosis marneffei in Thailand: report of five human cases. Am J Trop Med Hyg. 1984;33 (4):637-644. doi:10.4269/ajtmh.1984.33.637

11. Liyan X, Changming L, Xianyi Z, Luxia W, Suisheng X. Fifteen cases of penicilliosis in Guangdong, China. Mycopathologia. 2004;158(2):151-155. doi:10.1023/B:MYCO.0000041842.90633.86

12. Lam KY, Cheung F, Yam LY, Lee CH, Fung KH. Atypical manifestations in a patient with systemic lupus erythematosus. J Clin Pathol. 1997;50(2):174-176. doi:10.1136/jcp.50.2.174

13. Lo CY, Chan DT, Yuen KY, Li FK, Cheng KP. Penicillium marneffei infection in a patient with SLE. Lupus. 1995;4(3):229-231. doi:10.1177/096120339500400313

14. Ng GW, Cheuk W, Lee MK, Wu TC, Chau KF. Test and teach. Fever and disseminated lymphadenopathy in a SLE patient in Hong Kong. Diagnosis: penicilliosis. Pathology. 2006;38(4):353-355. doi:10.10 $80 / 00313020600820724$
15. Luo DQ, Chen MC, Liu JH, Li Z, Li HT. Disseminated Penicillium marneffei infection in an SLE patient: a case report and literature review. Mycopathologia. 2011;171(3):191-196. doi:10.1007/s11046010-9363-9

16. Kim HA, Jeon JY, An JM, Koh BR, Suh CH. C-reactive protein is a more sensitive and specific marker for diagnosing bacterial infections in systemic lupus erythematosus compared to S100A8/A9 and procalcitonin. J Rheumatol. 2012;39(4):728-734. doi:10.3899/ jrheum. 111044

17. Wong SY, Wong KF. Penicillium marneffei infection in AIDS. Patholog Res Int. 2011;2011:764293.

18. Brod SA. In MS: immunosuppression is passé. Mult Scler Relat Disord. 2020;40:10196. doi:10.1016/j.msard.2020.101967

19. Zeng W, Qiu Y, Lu D, Zhang J, Zhong X, Liu G. A retrospective analysis of 7 human immunodeficiency virus-negative infants infected by penicillium marneffei. Medicine (Baltimore). 2015;94 (34):e1439. doi:10.1097/MD.0000000000001439

20. Qiu Y, Pan M, Zhang J, et al. Two unusual cases of human immunodeficiency virus-negative patients with Talaromyces marneffei infection. Am J Trop Med Hyg. 2016;95(2):426-430. doi:10.4269/ ajtmh.15-0789

\section{Publish your work in this journal}

Infection and Drug Resistance is an international, peer-reviewed openaccess journal that focuses on the optimal treatment of infection (bacterial, fungal and viral) and the development and institution of preventive strategies to minimize the development and spread of resistance. The journal is specifically concerned with the epidemiology of antibiotic resistance and the mechanisms of resistance development and diffusion in both hospitals and the community. The manuscript management system is completely online and includes a very quick and fair peerreview system, which is all easy to use. Visit http://www.dovepress.com/ testimonials.php to read real quotes from published authors. 